\title{
The early Earth under a superflare and super-CME attack: prospects for life
}

\author{
Vladimir Airapetian ${ }^{1}$, Alex Glocer ${ }^{1}$ and Guillaume Gronoff ${ }^{2}$ \\ ${ }^{1}$ NASA Goddard Space Flight Center, \\ Greenbelt, MD, 20771 \\ email: vladimir.airapetian@nasa.gov \\ ${ }^{2}$ SSAI/NASA LaRC, Hampton, VA 23681 \\ email: guilaume.p.gronoff@nasa.gov
}

\begin{abstract}
Kepler observations suggest that G-type stars produce powerful flares suggesting that the early Earth may also have been exposed to frequent and energetic solar explosive events generated by the young Sun. We show that powerful coronal mass ejection (CME) events associated with superflares impacting the Earth magnetosphere with a frequency of 1 event/day. What was the impact of superflares, CMEs and associated solar energetic particle (SEPs) events on the atmospheric erosion of the young Earth and habitability? In this paper we discuss our three-dimensional (3D) magnetohydrodynamic (MHD) simulations that show that frequent and energetic CMEs from the early Sun continuously destroyed the sub-solar parts of Earth's magnetosphere at heights $<1.25 \mathrm{R}_{E}$. This suggests that CME shock accelerated energetic protons are capable of penetrating into the polar cap region and breaking atmospheric molecular nitrogen, the major ingredient of the early Earth atmosphere, into atomic nitrogen. Photo-collisional dissociation of molecular nitrogen and carbon dioxide creates reactive chemistry that efficiently produces nitrous oxide and hydrogen cyanide, the essential molecule in prebiotic life chemistry. This raises an possibility that frequent super-CMEs could serve as a potential catalyst for the origin of life on early Earth.
\end{abstract}

Keywords. Superflare, CME, SEP, Early Earth, Atmospheres, Prebiotic Chemistry

\section{Introduction}

Our Sun is a typical main-sequence cool star. Its dynamo driven magnetic field is a major source of magnetic activity producing solar explosive events (SEE) in its atmosphere. A powerful SEE is usually represented by three forms of activity including flares, coronal mass ejections (CME) and solar energetic particles (SEP). These processes energize the environment around the Sun and drive space weather that affect the Earth's magnetosphere and atmosphere. Specifically, solar flares produce a few hundred times increase in the XUV (X-ray and Extreme Ultraviolet) flux, which cause the photoionization of the terrestrial ionosphere and enhancement of its temperature and density. Earth-directed energetic CMEs cause strong perturbation of Earth's magnetosphere and induce large geomagnetic currents due to the enhanced wind dynamic pressure. The third form of activity, SEPs events, are characterized with proton accelerated by CME driven shocks to energies up to a few $\mathrm{GeV}$. Their penetration into the terrestrial atmosphere may cause chemical changes contributing to ozone destruction (Verkhodlyadova et al. 2015).

Recent observations of Kepler's field stars provided new insights on the role of paleo space weather from the young Sun. The data revealed that solar-types stars exhibit flare events with energies over 100 times greater than that observed on the Sun referred to as superflares (Shibayama et al. 2013). Flare statistics of such events suggests that the frequency of occurrence of superflares with energies $\geqslant 5 \times 10^{34}$ erg from G-type 
dwarfs follows a power-law distribution with spectral index $\sim 2.0$, which is comparable to those observed on dMe stars and the Sun. Specifically, young solar-like stars show the occurrence rate of superflares (with the energy $5 \times 10^{34} \mathrm{erg}$ ) at the rate of 0.1 events/day. This implies that the frequency of flares with the energy of $10^{33}$ ergs from the young Sun at $\leqslant 0.7$ Gyr is anticipated to be $\sim 250$ events/day (Airapetian et al. 2015a, 2016a). Because all powerful events from the current Sun are accompanied with CMEs and SEPs and those events propagate within a cone of $\geqslant 90 \mathrm{deg}$, we have concluded that powerful Earth-directed CMEs (super-CME or SCME events) and SEPs would produce a perfect storm (when the incoming interplanetary z-component of magnetic field is sheared with respect to the Earth's dipole magnetic field) at the rate of at least a few events per day. Because a CME event lasts for 2-3 days, this suggests that the Earth was under a quasi steady state impact from energetic CME and SEP events.

In this paper we discuss the effects associated with interaction of SCMEs and SEPs with the magnetosphere and lower atmosphere of the early Earth. We show that frequent and energetic solar explosive events may significantly impact chemistry, climate and habitability conditions on the early Earth, Mars and exoplanets around M dwarfs.

\section{Effects of Superflares From The Young Sun}

XUV (SXR and EUV) radiation induced by frequent and energetic flare activity from the young Sun could directly impact planetary atmospheric pressure and its climate through the erosion of the planetary atmospheres (Kulikov et al. 2007; Lundin et al. 2007). We have derived the spectral energy distribution (SED) in the XUV band using the EVE/SDO data for the X5.4 solar flare occurred on March 7, 2012. The Vacuum Ultraviolet contribution of the total radiative output is obtained by implementing the Flare Irradiance Spectral Model (FISM) that estimates the solar irradiance at wavelengths from 1 to $190 \mathrm{~nm}$ (Chamberlin et al. 2008). In Airapetian et al. 2016b, we compared the reconstructed XUV spectrum for a typical M1 dwarf, GJ 832, scaled to the distance its Super-Earth planet (0.16 AU) with the XUV flux from the young, the current Sun and a typical X5 solar flare scaled at 1 AU.

Figure 1 presents the SED of the X5.4 solar flare (blue), the M 1 dwarf quiescent flux (red), the XUV fluxes from the young Sun at 0.7 Ga (solid orange) and the current quiet Sun (dashed orange). The figure shows that the solar flare, M dwarf and the young Sun have comparable XUV flux and the shape of SED at wavelength shorter than Ly- $\alpha$. Their fluxes are from 10-1000 times greater than the flux from the current quiet Sun. This plot suggests that the XUV flux from the quiescent emission from both $\mathrm{M}$ dwarf and the young Sun is dominated by frequent and energetic X-type flare emission. This is consistent with our estimates of frequency of occurrence of large flares derived from the Kepler data (see Section 1).

In order to model the response of the planetary atmospheric dynamics to such large XUV fluxes, we used the Polar Wind Outflow Model (PWOM) model coupled with SuperThermal Electron Transport (STET) developed by Glocer et al. (2012) and Khazanov (2011). The PWOM code solves the equations of continuity, momentum conservation and energy conservation for $\mathrm{O}^{+}, \mathrm{H}^{+}$and $\mathrm{He}^{+}$. Photoelectrons produced due to photoionization of atmospheric oxygen, nitrogen, hydrogen and helium propagate upward along the magnetic field lines in the polar cap region. They outrun ions because of their inertia resulting in charge separation between the electrons and ions. The produced polarization electric field is derived from the electron momentum equation. A separate energy equation is used to evolve the electron temperature. Neutral $\mathrm{N}_{2}, \mathrm{O}_{2}, \mathrm{O}$ and $\mathrm{H}$ are treated as a static fixed atmosphere that reacts collisionally and chemically with the 


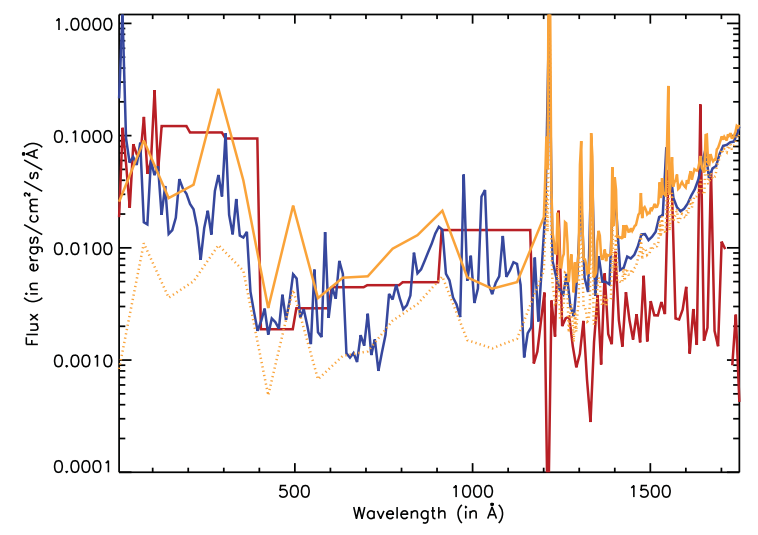

Figure 1. The SED in the XUV band for the solar X5.4 flare (blue) \& the young Sun SED (orange) scaled to $1 \mathrm{AU}$ and GJ $832 \mathrm{SED}$ (red) scaled to $0.16 \mathrm{AU}$.
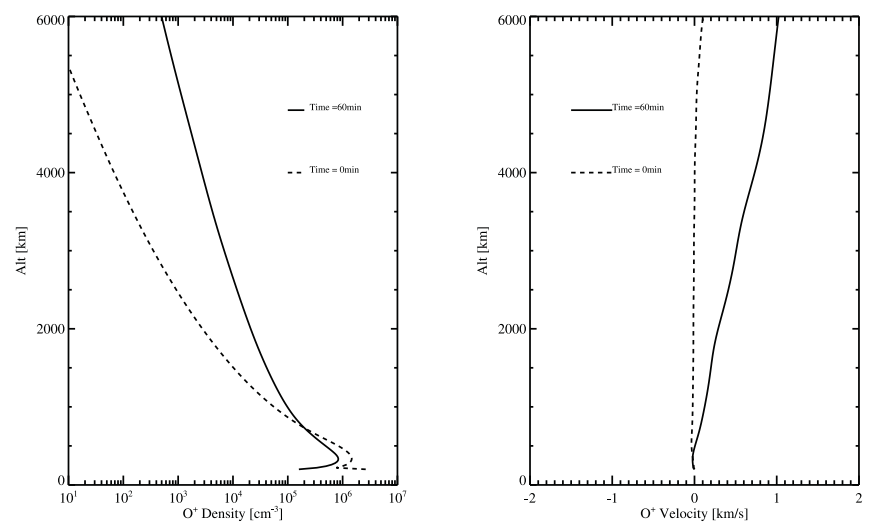

Figure 2. The radial density (left panel) and velocity (left panel) profiles of the $\mathrm{O}^{+}$ions in the Earth atmosphere.

ions, including such effects as photoionization and charge exchange. This code calculates the produced mass outflow of hydrogen and oxygen ions in the Earth atmosphere.

We applied the WPOM + STET model for the case of the young Sun's XUV flux presented in Figure 1. Figure 2 shows the steady state solution of the density (left panel) and velocity (right panel) of $\mathrm{O}^{+}$ions between $200-6000 \mathrm{~km}$ along a single magnetic flux line in the polar regions of Earth.

Our model suggests that the increase of the XUV flux by a factor of 10 enhances the rate of the outflow by a factor of 30 . However, our current model does not include the impact ionization by precipitating electrons formed due to reconnection events ignited by magnetospheric compression due to the impact from a CME event associated with a superflare. Empirical data (Wei et al. 2013) suggest that precipitating electrons formed during magnetospheric storms contribute significantly to the mass loss rate. Thus, our results may suggest that the enhanced XUV flux from the early Sun could contribute significantly to the total atmospheric loss from the early Earth due non-thermal processes. 


\section{Magnetospheric Models of the Paleo Solar Wind and CME events}

\subsection{Properties of the Paleo Solar Wind}

Paleo solar wind could play an important role in atmospheric erosion (Lundin et al. 2007). We have recently reconstructed the properties of the paleo solar wind by using a 3D MHD simulation code, ALF3D, previously validated for the conditions of the current solar wind (Airapetian \& Usmanov 2015b). The code solves ideal time-dependent compressible MHD equations for electrons. ions and pickup protons under the assumption of a dipole magnetic filed in the co-rotating with the Sun's frame of reference. The magnetic dipole is aligned with the rotation axis. For each of the three simulations representing the solar wind at $0.7 \mathrm{Ga}, 2 \mathrm{Ga}$ and $4.6 \mathrm{Ga}$, we assume that the wind is driven by combination of the thermal pressure gradient and Alfvén waves generated at the wind base. We used the empirical scaling for the surface magnetic field with the star's age, observational constrains for the coronal wind base density \& temperature, the magnetic field and the Alfvén wave amplitudes as inputs for the MHD model. Our simulations suggest that the velocity of fast wind emanating from the polar open magnetic field regions of the young Sun (at $0.7 \mathrm{Ga}$ ) is about twice greater $(1318 \mathrm{~km} / \mathrm{s})$ and the mass loss rate could be over 80 times greater than the mass loss rates from the current Sun. Thus, these results imply that the dynamic pressure from the paleo solar wind was over 160 times greater than the current value and are consistent with other models and the rates derived from observations of young stars (Wood et al. 2006; Cranmer and Saar 2011; Sterenborg et al. 2011).

\subsection{Extreme CME Interaction With The Magnetosphere of Early Earth}

To model the effects of the paleo solar wind (see Section 2) combined with its perturbation due to propagating super CME from the young Sun on the Earth's magnetosphere and ionosphere, we utilized a single-fluid, time dependent fully non-linear 3D MHD code BATSRUS for fully ionized plasma. This code is coupled to Rice Convection Model (RCM, de Zeeuw et al. 2004). The MHD part of the code calculates the dynamic response of the large-scale magnetospheric plasma to varying solar wind conditions in a self consistent manner by using the block-adaptive wind Roe-type upwind scheme global MHD code. The magnetospheric currents near the inner boundary of the MHD simulation are mapped to the ionosphere. A potential solver is then used which combines these currents with a conductance map of the ionosphere (including solar and auroral contributions) to produce the electric potential in the ionosphere. This potential is then used to set the electric field and corresponding drift at the magnetospheric simulations inner boundary.

Here we describe the scenario of the super CME (SCME) induced perfect magnetospheric storm, when the incoming cloud magnetic field, Bz component is sheared with respect to the Earth's magnetic field. To model a SCME event, we used the model of a paleo solar wind described in Section 2.1 and the physical parameters of a Carrington-type event with the energy of $2 \times 10^{33} \mathrm{erg}$. We used the current value of the magnetic moment of the Earth's magnetosphere. Figure 1 presents a 2D map of the steady-state plasma density superimposed by magnetic field lines for the magnetospheric con-figuration in the $\mathrm{Y}=0$ plane corresponding to the initial 30 minutes of the simulations, when the Earth's magnetosphere was driven only by dynamic pressure from the paleo solar wind. Figure 3 shows the state of the magnetosphere two hours later when the CME cloud hits the Earth magnetosphere. At this time, the solar wind dynamic pressure and the magnetic reconnection between the southward directed CME's cloud magnetic field and 

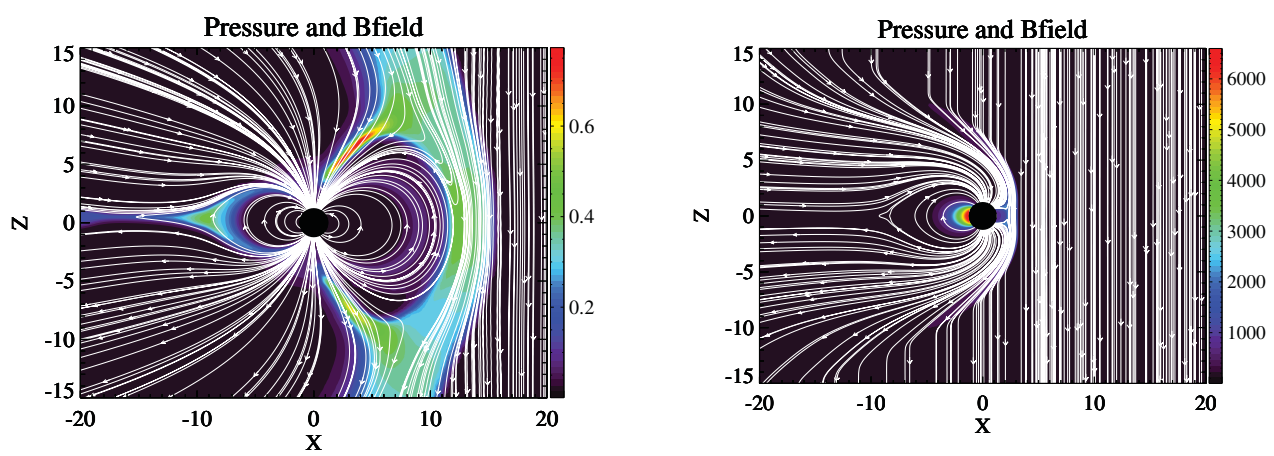

Figure 3. The initial (left panel) and final solution (right panel) for the magnetospheric structure driven by a SCME impact on the early Earth magnetosphere (Airapetian et al. 2015).

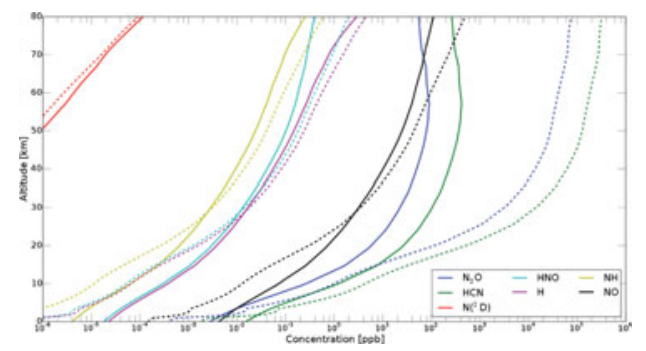

Figure 4. The radial profile of the steady state mixing ratios of atmospheric species forming as a result of photo-collisional processes in the early terrestrial atmosphere driven by an SEP event (Airapetian et al. 2016a).

northward Earth's dipole magnetospheric field pushes the dayside magneto-sphere earthward reducing the stand-off distance from 9 to 1.5 Earth's radii.

The generated convective electric field, $\vec{E}=\vec{V} \times \vec{B}$, drives large field aligned current and produces significant disturbance of the magnetospheric field shifting the boundary of the open-closed field shifts to $36 \mathrm{deg}$ in latitude and producing a polar cap opening to $70 \%$ of the Earth's dipole magnetic field. Energetic particles accelerated in a CME driven shocks can then efficiently penetrate the early terrestrial atmosphere through the expended polar cap region. The ionospheric Joule heating rate in the polar cap region is $4000 \mathrm{erg} / \mathrm{cm}^{2} / \mathrm{s}$. This heating drives the thermal pressure gradient and produces a polar wind with the mass loss of $20 \mathrm{~kg} / \mathrm{s}$ of hydrogen plasma or 20 times of the current value.

\section{Chemical Impact of Paleo SEP events}

To study the effects of a strong SEP event associated with a SCME event, we used our Aeroplanet model to simulate the atmospheric chemistry of the highly reduced nitrogendominated ( $93 \%$ of $\mathrm{N}_{2}, 6 \%$ of $\mathrm{CO}_{2}$ and less than $1 \%$ of $\mathrm{H}_{2} \mathrm{O}$ and $\mathrm{CH}_{4}$ ) prebiotic Earth's atmosphere at a surface pressure of 1 bar. The upper boundary of the atmosphere at $100 \mathrm{~km}$ is exposed to the EUV-XUV flux with the spectrum reconstructed for the early Sun at 3.8 Gyr and to the of energetic protons within the energy range $1-1000 \mathrm{MeV}$ with the energy flux 50 times of Jan 20, 2005 SEP event.

The model calculates photoabsorption of the EUV-XUV flux from the early Sun and particle (electron and proton) fluxes to compute the corresponding fluxes at all atmospheric altitudes between $200 \mathrm{~km}$ to the surface. These fluxes are used to calculate 
the photo and particle impact ionization/dissociation rates of the atmospheric species producing secondary electrons due to ionization processes. Then, using the photon flux and the photoionization-excitation-dissociation cross-sections, the model calculates the production of ionized and excited state species and as a result, photoelectrons. In this steady-state model, energetic protons from an SEP event produce ionizations, dissociations, dissociative ionizations, and excitations in the middle and low atmosphere of Earth. The penetrating protons produce secondary energetic electrons due to impact ionization that efficiently destroy molecular nitrogen into odd nitrogen, $\mathrm{N}(2 \mathrm{D})$ and $\mathrm{N}(4 \mathrm{~S})$ and subsequent destruction of carbon dioxide and methane. These processes produce $\mathrm{NO}_{x}(\mathrm{~N}$, $\mathrm{NO}, \mathrm{NO}_{2}$ ), $\mathrm{CO}, \mathrm{CH}$ and $\mathrm{NH}$ constituents in the polar regions of atmosphere. Our chemical model includes 120 neutral chemical reactions. The results suggest that the reaction of $\mathrm{NO}$ with $\mathrm{NH}$ is efficient in producing nitrous oxide in the low atmosphere. Nitrous oxide is a powerful greenhouse gas and its abundance at the level of tens ppbm and can explain the Young Sun's paradox for the early Earth (see Figure 4).

\section{Implications for Life on Early Earth}

Our models discussed in this paper suggest that the paleo space weather could play a crucial role in the magnetospheric and atmospheric dynamics of the early Earth and possibly Mars. First, XUV fluxes from the young Sun produced by X-type flares can enhance the mass loss from the early Earth atmosphere by a factor of $>30$. Second, energetic CMEs propagating on top of the dense and fast paleo solar wind produce large (by a factor of 160) dynamic pressure that compresses the magnetosphere to $1.25 \mathrm{R}_{E}$, then opens $70 \%$ of the Earth magnetic field and deposits large Joule heating that drives a polar outflow. Third, an SEP event associated with a SCME may produce high energy protons that form secondary electrons due to collisional ionization, and in turn also contribute to the dissociation, ionization and excitation of atoms and molecules in the low atmosphere of Earth. Specifically, collision driven nitrogen fixation is an essential process that ignites reactive chemistry in the low atmosphere that efficiently produces nitrous oxide and complex organic chemistry. The proposed models of paleo space weather events can be applicable for young active solar-like stars and $M$ dwarfs. The effects of extreme space weather challenge the definition of the habitability zone if high energy processes from host stars including XUV emission and the wind/CME dynamic pressure could contribute to reduction of the atmospheric pressure of exoplanets to the level of $\leqslant 0.01$ bar over a few hundred million years. Then, this will prevent the existence of liquid water at any temperature. Further development of such models are required to characterize the climate and habitability conditions imposed by extreme space weather.

\section{References}

Airapetian, V., Glocer, A., Gronoff, G., Hebrard, E., Danchi, W 2016a, Nature Geoscience, accepted.

Airapetian, V., Glocer, A., Khazanov, G., Parker, L., France, K. 2016b, submitted.

Airapetian, V., \& Glocer, A. and Danchi, W. 2015a, In the 18th Cambridge Workshop on Cool Stars, Stellar Systems, and the Sun Proceeding of Lowell Observatory (9-13 June 2014) Edited by G. van Belle and H. Harris

Airapetian, V. and Usmanov, A. 2015b, ApJ Letters, 817, L24

\&Chamberlin, P. C.,Woods, T. N., Eparvier, Francis G. 2008, Space Weather, Volume 6, Issue 5, CiteID S05001

Claire, M. W., Sheets, J. Cohen, M., Ribas, I., Meadows, V. S., \& Catling, D. C. 2012, ApJ, 57, 12 
Cranmer, S. R. \& Saar, S. H. 2011 ApJ, 741, 23

de Zeeuw, D. L., Sazykin, S., Wolf, R. A., Gombosi, T. I., Ridley, A. J., \& Toth, G. 2004, JGR, 109, A12

Glocer, A., Kitamura, N., Toth, G., \& Gombosi, T. 2012, JGR, 117, Issue A4, CiteID A04318

Gough, G. O. 1981, Solar Physics, 74, 21

Khazanov, G. V. 2011. Kinetic Theory of Inner Magnetospheric Plasma. Springer: New York, 372,584

Kulikov, Y. N., Lammer, H., Lichteneg-ger, Herbert I. M.; Penz, T. Breuer, D.,Spohn, T., Lundin, R., \& Bienat, H. K., 2007, Space Science Reviews, 129, Issue 1-3, 207

Lundin, R., Lammer, H., \& Ribas, I. 2007, 129, Issue 1-3, 245

Sterenborg, M. G., Cohen, O. , Drake, J. J., \& Gombosi, T. I. 2011, 116, A01217.

Verkhoglyadova, Wang, S., Mlynczak, M. G., \& Hunt, L. A. and Zank, G. P. 2015, JGR, 120, 12

Wei, Y. and 13 co-authors 2012, JGR, 117, Issue A3, A03208

Wood, B. E., Mller, H-R, Zank, G. P., Linsky, J. L., \& Redfield, S. 2005, ApJ, 628, L143. 